\title{
Das neue fib Bulletin 80 - Teilsicherheitsbeiwerte für die Nachrechnung bestehender Massivbauwerke
}

Die zuverlässigkeitsbasierte Nachrechnung bestehender Tragwerke kann auf Basis verschiedener wissenschaftlicher Verfahren erfolgen. Da jedoch bisher nur wenige mit den Eurocodes kompatible Verfahren für eine praxisgerechte Anwendung existieren, kommen für die Nachrechnung bestehender Tragwerke meist semiprobabilistische Verfahren und Teilsicherheitsbeiwerte zur Anwendung, wie sie auch zur Bemessung von Neubauwerken verwendet werden. Eine solche Beurteilung bzw. Nachrechnung bestehender Tragwerke führt oft zu sehr konservativen Ergebnissen, verbunden mit aufwendigen und kostenintensiven Verstärkungsmaßnahmen.

Vor diesem Hintergrund wurde in der fib Task Group 3.1 "Reliability and safety evaluation: full-probabilistic and semi-probabilistic methods for existing structures" das neue Bulletin 80 „Teilsicherheitsbeiwerte für die Nachrechnung bestehender Massivbauwerke“ [1] erarbeitet, welches sowohl Empfehlungen für die Teilsicherheitsbeiwerte der Einwirkungs- als auch der Widerstandsseite enthält.

Im vorliegenden Beitrag werden neben zwei Verfahren zur Bestimmung modifizierter Teilsicherheitsbeiwerte für die Nachrechnung bestehender Massivbauwerke nach fib Bulletin 80 [1] mit schwerpunktmäßiger Betrachtung der Widerstandsseite auch Methoden zur Anpassung des Zielzuverlässigkeitsindex nach fib Bulletin 80 [1] vorgestellt. Darüber hinaus wird das Vorgehen an einem Anwendungsbeispiel erläutert.

1

\section{Einleitung}

Grundsätzlich gewinnt das Bauen im Bestand zunehmend an Bedeutung. Werden infolge Änderung, Umnutzung oder Instandsetzung statische Nachrechnungen an bestehenden Tragwerken in Stahlbetonbauweise erforderlich, so sind diese basierend auf charakteristischen Materialkennwerten nach den aktuell gültigen Regelwerken, d.h. den Eurocodes, zu führen. Dieses Vorgehen führt in vielen Fällen zu einer sehr konservativen Beurteilung der bestehenden Tragstruktur, da die in den Eurocodes enthaltenen Regelungen ausschließlich auf die Bemessung von Neubauwerken ausgelegt sind und somit nicht die Besonderheiten bestehender Tragwerke berücksichtigen.

Während für die Bestimmung charakteristischer Materialkennwerte für die Nachrechnung bestehender Massivbauwerke in Deutschland u.a. mit den DAfStb-Heften 616 [2] und 619 [3], den DBV-Merkblättern Bauen im Bestand „Beton und Betonstahl“ [4] und „Bewertung der In-situ-Druckfestigkeit“ [5] sowie der Nachrechnungs-
Partial factor methods for existing concrete structures In literature different methods for the reliability-based assessment of existing concrete structures have been developed. Nevertheless, a consistent reliability-based assessment framework and a practically applicable codified approach which is compatible to the Eurocodes and accessible for common structural engineering problems in everyday practice is still missing. Currently the assessment of existing structures is mostly based on simplified procedures based on the partial factor method, commonly applied for the design of new structures. Such an assessment of an existing structure may lead to conservative results and expensive rehabilitation and strengthening measures.

Therefore, the fib Task Group 3.1 "Reliability and safety evaluation: full-probabilistic and semi-probabilistic methods for existing structures" developed the new fib Bulletin 80 [1] "Partial factor methods for existing concrete structures". The new Bulletin 80 includes modified partial factors for materials and actions.

In the present contribution, based on fib Bulletin 80 [1], two methods for the modification of partial factors for the assessment of existing structures are presented. Furthermore, different methods for the adaption of the target reliability index are described. Finally, the approach is explained, using an example.

richtlinie [6] bereits verschiedene Regelwerke zur Verfügung stehen, liegt für die Festlegung der für die Nachrechnung erforderlichen Teilsicherheitsbeiwerte vor allem das DBV-Merkblatt Modifizierte Teilsicherheitsbeiwerte für Stahlbetonbauteile [7] vor. Die dort enthaltenen Verfahren beruhen im Wesentlichen auf der Bestimmung der tatsächlichen Streuung der am Bestandsbauwerk vorliegenden Materialien.

Auf internationaler Ebene wurde mit dem neuen fib Bulletin 80 [1] (Bild 1) in der fib Task Group 3.1 ein Bericht erarbeitet, der bei der Festlegung des einzuhaltenden $\mathrm{Zu}$ verlässigkeitsniveaus Aspekte der menschlichen Sicherheit sowie wirtschaftliche Überlegungen enthält.

Wie in Eurocode 0 wird auch in [1] Bezug auf Schadensfolgeklassen und die Restlebensdauer genommen. Die fachliche Diskussion, inwieweit für Bestandstragwerke solche oder vergleichbare Kriterien im bauaufsichtlich geregelten Bereich Berücksichtigung finden sollen, steht national noch aus. Die aktuellen, von CEN/TC 250/ WG2.T1 erarbeiteten Entwürfe zur Fortschreibung von 


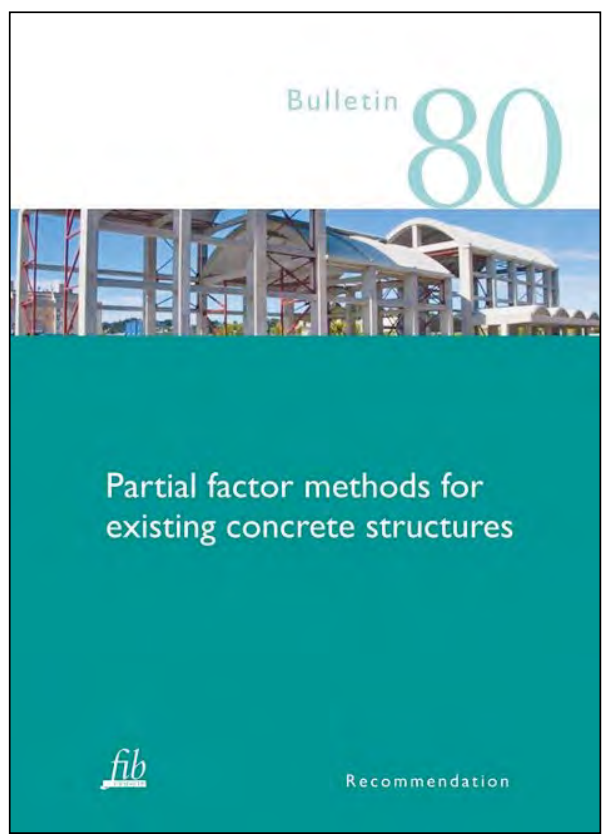

Bild 1 fib Bulletin 80 - Teilsicherheitsbeiwerte für die Nachrechnung bestehender Massivbauwerke, fib Task Group 3.1 [1]

fib Bulletin 80 - Partial factor methods for existing concrete structures, Recommendation, fib Task Group 3.1 [1]

Eurocode 0 auf bestehende Tragwerke sehen hierzu jeweils nationale Festlegungen vor. Grundsätzlich stehen die im Bulletin 80 aufgeführten Verfahren nicht im Widerspruch zu den im DBV-Merkblatt Modifizierte Teilsicherheitsbeiwerte für Stahlbetonbauteile [7] enthaltenen Ansätzen, sondern sind vielmehr als Ergänzung zu sehen.

Für die praktische Anwendbarkeit werden in [1] mit der „Design value method“ (DVM) und der „Adjusted partial factor method" (APFM) zwei Verfahren zur Festlegung modifizierter Teilsicherheitsbeiwerte für die Nachrechnung bestehender Massivbauwerke vorgeschlagen. Beide Verfahren ermöglichen die Anwendung individuell angepasster Werte des Zielzuverlässigkeitsindex. Die DVM ermöglicht eine direkte Berechnung von Teilsicherheitsbeiwerten; die APFM schlägt hingegen Anpassungsfaktoren vor, mit denen die für den Neubau nach Eurocode gültigen Teilsicherheitsbeiwerte modifiziert bzw. angepasst werden können. Die jeweiligen Anwendungsbereiche werden in [1] erläutert.

Grundsätzlich ist zu beachten, dass die in [1] beschriebenen Verfahren lediglich für die Nachrechnung nicht geschädigter Tragwerke verwendet werden dürfen. In den nachfolgenden Abschnitten werden die wesentlichen Ansätze aus dem fib Bulletin 80 [1] wiedergegeben.

\section{Modifizierte Zielzuverlässigkeitsindizes für die Nachrechnung bestehender Tragwerke Allgemeines}

Die Nachrechnung bestehender Tragwerke unterscheidet sich von der Bemessung von Neubauwerken in vielerlei Hinsicht. Wesentliche Aspekte sind:
- Das Erreichen eines vorgegebenen Zuverlässigkeitsniveaus verursacht bei der Nachrechnung einen größeren Kostenanstieg als im Neubaufall.

- Die Restnutzungsdauer bestehender Tragwerke ist oft kleiner als die der Bemessung zugrunde liegende geplante Nutzungsdauer von 50 bis 100 Jahren.

- Für die Nachrechnung bestehender Tragwerke können zusätzliche Informationen zum tatsächlichen Bauwerkszustand aus einer Bauwerksuntersuchung vorliegen.

In EN 1990:2002 [8] sind verschiedene Verfahren zur Kalibrierung von Teilsicherheitsbeiwerten enthalten. Ein wesentliches Verfahren ist dabei die „First Order Reliability Method“ (FORM). Als Bemessungswerte $R_{\mathrm{d}}$ und $E_{\mathrm{d}}$ werden dabei die Werte der Variablen $R$ und $E$ am FORM-basierten Bemessungspunkt gewählt. Als Maß der Zuverlässigkeit wird dabei der Zuverlässigkeitsindex $\beta$ verwendet. Der Zusammenhang zwischen Zuverlässigkeitsindex und Versagenswahrscheinlichkeit kann mithilfe der standardisierten Normalverteilung gemäß Gl. (1) beschrieben werden. In Tab. 1 sind exemplarisch einige Werte dargestellt.

$$
\beta=-\Phi^{-1}\left(P_{\mathrm{f}}\right)
$$

In Deutschland finden bei der Nachrechnung bestehender Tragwerke wie auch bei der Bemessung von Neubauwerken die Differenzierung in verschiedene Schadensfolgeklassen, wirtschaftliche Optimierung sowie probabilistische Analysen der Bauwerkszuverlässigkeit bisher meist keine Anwendung. Dennoch werden in den Abschn. 2.2 und 2.3 die in fib Bulletin 80 [1] enthaltenen Verfahren zur Bestimmung des Zielzuverlässigkeitsindex $\beta$ erläutert, um Vorteile und Zugewinne durch derartige Verfahren aufzuzeigen.

Die Wahl des Zielzuverlässigkeitsindex hat keine Auswirkung auf die Anwendbarkeit der in Abschn. 3 vorgestellten Verfahren zur Anpassung der Teilsicherheitsbeiwerte für die Nachrechnung bestehender Tragwerke, da dort der Zielzuverlässigkeitsindex als variable Größe berücksichtigt werden kann. Folglich können die in [1] beschriebenen Verfahren auch ohne Berücksichtigung verschiedener Schadensfolgeklassen angewendet werden.

Die Schaffung einer baurechtlichen Basis, welche die allgemeine Anwendung der nachfolgend erläuterten Verfahren zur Festlegung des Zielzuverlässigkeitsindex in Deutschland gestattet, steht aktuell noch aus.

\subsection{Wirtschaftliche Überlegungen}

In Hinblick auf Betrachtungen der Wirtschaftlichkeit sollen nach [1] die Zielzuverlässigkeitsindizes unter Berücksichtigung der Bauwerkskosten und des zugehörigen Risikos, also dem Produkt aus Versagenswahrscheinlichkeit und den direkten und indirekten Kosten eines Versagens, gewählt werden. Allgemein führt die 
Tab. 1 Beziehung zwischen $\beta$ und $P_{\mathrm{f}}$ Probability of failure $P_{\mathrm{f}}$ and reliability index $\beta$

\begin{tabular}{llllllll}
\hline$P_{\mathrm{f}}$ & $10^{-1}$ & $10^{-2}$ & $10^{-3}$ & $10^{-4}$ & $10^{-5}$ & $10^{-6}$ & $10^{-7}$ \\
$\beta$ & 1,3 & 2,3 & 3,1 & 3,7 & 4,2 & 4,7 & 5,2 \\
\hline
\end{tabular}

Umsetzung höherer Anforderungen an das Zielzuverlässigkeitsniveau im Rahmen der Nachrechnung und der daraus ggf. resultierenden Verstärkungsmaßnahmen zu höheren Kosten als die Umsetzung der gleichen Anforderungen bei Neubauwerken. Das heißt, der in Hinblick auf eine wirtschaftliche Optimierung bei der Nachrechnung $\mathrm{zu}$ wählende Zuverlässigkeitsindex darf kleiner sein als der im Neubaufall zu verwendende Wert. Die Aufrechterhaltung des bestehenden Zustands des Bestandstragwerks unter Berücksichtigung einer erhöhten Versagenswahrscheinlichkeit stellt nach [1] die wirtschaftlich optimale Entscheidung dar.

In [1] wird empfohlen, das Bestandstragwerk zunächst anhand des Mindestzielzuverlässigkeitsindex $\beta_{0}$ zu bewerten und erst dann ggf. so zu ertuichtigen, dass nach der Ertüchtigung der Zielzuverlässigkeitsindex $\beta_{\text {up }}$ erreicht wird. Für den im Grenzzustand der Tragfähigkeit (GZT) anzusetzenden Mindestzielzuverlässigkeitsindex $\beta_{0}$, unterhalb dessen das Bauwerk ertüchtigt werden sollte, darf nach [1] der im Neubaufall für die geplante Nutzungsdauer anzusetzende Zielzuverlässigkeitsindex allgemein um $\Delta \beta=1,5$ reduziert werden. Individuelle wirtschaftliche Optimierungen können im Einzelfall zu noch größeren Werten für $\Delta \beta$ führen. Für den aktualisierten Zielzuverlässigkeitsindex $\beta_{\text {up }}$ darf nach [1] der im Neubaufall für die geplante Nutzungsdauer anzusetzende Zielzuverlässigkeitsindex allgemein um $\Delta \beta=0,5$ reduziert werden.

In Tab. 2 sind die für eine wirtschaftliche Optimierung im Grenzzustand der Tragfähigkeit (GZT) anzusetzenden Mindestwerte des Zielzuverlässigkeitsindex zusammengefasst. Die Ausgangswerte $\beta$ entsprechen dabei den in EN 1990:2002 [8] enthaltenen Werten. Betrachtet man lediglich eine wirtschaftliche Optimierung, so dürfen nach [1] die in Tab. 2 angegebenen Werte für den Zuverlässigkeitsindex $\beta$ unabhängig vom Bezugszeitraum (Restnutzungsdauer) verwendet werden.

Der in Tab. 2 für die Schadensfolgeklasse CC 2 infolge einer wirtschaftlichen Optimierung angegebene Wert $\beta_{\text {up }}=3,3$ entspricht nach DBV-Heft 24 [9] in etwa den Grundlagen des DBV-Merkblatts Modifizierte Teilsicherheitsbeiwerte für Stahlbetonbauteile [7]. Den dort angegebenen Teilsicherheitsbeiwerten liegt ein Zielzuverlässigkeitsindex von 3,2 zugrunde.

Bei der Festlegung des erforderlichen Zielzuverlässigkeitsindex sind nach [1] neben der wirtschaftlichen Optimierung auch die im nachfolgenden Abschnitt beschriebenen Anforderungen an die Zuverlässigkeit der Tragstruktur zu betrachten. Der höhere Wert ist für die Nachrechnung zu verwenden.
Tab. 2 Zuverlässigkeitsindex für die Nachrechnung bestehender Tragwerke im GZT für die Restnutzungsdauer - wirtschaftliche Optimierung [1] Reliability index for existing structures related to ultimate limit states and remaining working life, economic optimisation [1]

\begin{tabular}{llll}
\hline $\begin{array}{l}\text { Schadensfolge- } \\
\text { klassen }\end{array}$ & $\boldsymbol{\beta}$ & $\boldsymbol{\beta}_{\text {up }}$ & $\boldsymbol{\beta}_{\mathbf{0}}$ \\
\hline CC 3 & 4,3 & $4,3-0,5=3,8$ & $4,3-1,5=2,8$ \\
CC 2 & 3,8 & $3,8-0,5=3,3$ & $3,8-1,5=2,3$ \\
CC 1 & 3,3 & $3,3-0,5=2,8$ & $3,3-1,5=1,8$ \\
\hline
\end{tabular}

\subsection{Betrachtung der menschlichen Sicherheit}

Stellen die Anforderungen an die menschliche Sicherheit das maßgebende Kriterium dar, ist allgemein eine über die Nutzungsdauer konstante jährliche Versagenswahrscheinlichkeit zu verwenden. Nach ISO 2394:1998 [10] liegt in entwickelten Ländern die Wahrscheinlichkeit, im Alltag infolge eines Unfalls zu sterben, bei ca. $10^{-4}$ pro Jahr. Eine Sterbewahrscheinlichkeit für Opfer eines Bauwerkversagens, die größer als die Sterbewahrscheinlichkeit infolge eines Alltags-Unfalls ist, findet gesellschaftlich keine Akzeptanz. Daher finden sich in der Literatur für das Einzelrisiko (Individual risk $=$ IR) Werte zwischen $10^{-5}$ und $10^{-6}$ pro Jahr. Für die in [1] dargestellten Empfehlungen wird ein Einzelrisiko von $10^{-5}$ pro Jahr verwendet.

Basierend auf dem Einzelrisiko kann der Zielwert der Versagenswahrscheinlichkeit $P_{\mathrm{ft}, \mathrm{IR}}$ in Abhängigkeit von der Wahrscheinlichkeit von Opfern $P_{\mathrm{c|f}}$ nach Gl. (2) berechnet werden.

$$
P_{\mathrm{ft}, \mathrm{IR}} \leq 10^{-5} / P_{\mathrm{c} / \mathrm{f}}
$$

In Hinblick auf Verluste von Menschenleben unterscheidet EN 1990:2002 [8] zwischen den Schadensfolgeklassen CC 1 (Low), CC 2 (Medium) und CC 3 (High). Die von STEENBERgEN und VROUWENVELDER [11] bestimmte zugehörige Wahrscheinlichkeit von Opfern bei Bauwerksversagen $P_{\mathrm{c} \mid \mathrm{f}} *$ ist in Tab. 3 dargestellt. Zusätzlich ist in Tab. 3 neben den in fib Bulletin 80 [1] gewählten Werten auch die Wahrscheinlichkeit von mindestens einem Opfer bei Versagen des Tragwerks nach ElDUKAIR und АYYUв [12] dargestellt.

Unter Berücksichtigung der in Tab. 3 angegebenen Werte können mit Gl. (2) die Zielwerte der jährlichen Versagenswahrscheinlichkeit eines Bauteils wie folgt berechnet werden:

$$
\begin{array}{lll}
\text { CC 3: } & P_{\mathrm{ft}, \mathrm{IR}} \leq 10^{-5} / 0,20=5 \cdot 10^{-5} & \beta_{\mathrm{t}, \mathrm{IR}} \geq 3,9 \\
\mathrm{CC} 2: & P_{\mathrm{ft}, \mathrm{IR}} \leq 10^{-5} / 0,05=2 \cdot 10^{-4} & \beta_{\mathrm{t}, \mathrm{IR}} \geq 3,6 \\
\mathrm{CC} 1: & P_{\mathrm{ft}, \mathrm{IR}} \leq 10^{-5} / 0,01=10^{-3} & \beta_{\mathrm{t}, \mathrm{IR}} \geq 3,1
\end{array}
$$

Die zugehörigen Zielwerte des Zuverlässigkeitsindex $\beta_{\mathrm{t}, \mathrm{IR}}$ wurden mit dem in EN 1990:2002 [8] beschriebenen 
Tab. 3 Wahrscheinlichkeit von Todesopfern bei Bauwerksversagen [1] Probabilities of casualty given structural failure [1]

\begin{tabular}{|c|c|c|c|c|}
\hline 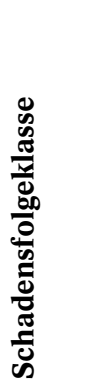 & 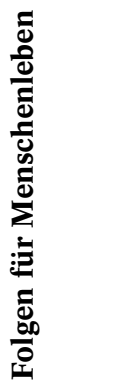 & $e^{*_{U}^{*}}$ & 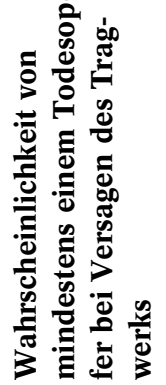 & 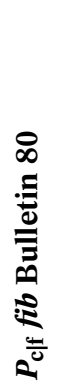 \\
\hline $\mathrm{CC} 3$ & Hoch & 0,3 & $0,03-0,055$ & 0,20 \\
\hline CC 2 & Mittel & 0,03 & $0,01-0,03$ & 0,05 \\
\hline CC 1 & Niedrig & 0,001 & 0,005 & 0,01 \\
\hline
\end{tabular}

Zusammenhang zwischen Versagenswahrscheinlichkeit und Zuverlässigkeitsindex berechnet. Angepasst auf einen Bezugszeitraum $t_{\text {ref }}$ ergeben sich die nachfolgenden Werte:

$$
\begin{array}{ll}
\text { CC 3: } & P_{\mathrm{ft}, \mathrm{IR}} \leq t_{\mathrm{ref}} \cdot 5 \cdot 10^{-5} \\
\text { CC 2: } & P_{\mathrm{ft}, \mathrm{IR}} \leq t_{\mathrm{ref}} \cdot 2 \cdot 10^{-4} \\
\mathrm{CC} 1: & P_{\mathrm{ft}, \mathrm{IR}} \leq t_{\mathrm{ref}} \cdot 10^{-3}
\end{array}
$$

Unter Berücksichtigung des Einzelrisikos (Individual risk $=$ IR) kann somit der Zielzuverlässigkeitsindex als Funktion des Bezugszeitraums angegeben werden. In Bild 2 ist der Zielzuverlässigkeitsindex $\beta_{\mathrm{t}, \mathrm{IR}}$ in Abhängigkeit vom

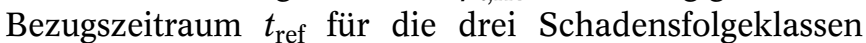
CC 1-3 angegeben. Weiterführende Ansätze zur Berücksichtigung einer Restnutzungsdauer bei der Festlegung des Zielzuverlässigkeitsindex können u.a. [13] entnommen werden.

Über die Betrachtung des Einzelrisikos hinaus werden im fib Bulletin 80 [1] auch noch verschiedene Ansätze zur Berücksichtigung der möglichen Anzahl der Opfer im Falle eines Versagens sowie Konzepte zur Kombination von wirtschaftlichen Aspekten und Anforderungen an die Sicherheit beschrieben.

\section{Teilsicherheitsbeiwerte für die Nachrechnung bestehender Massivbauwerke \\ 3.1 Allgemeines}

Für die Bemessung (Neubau) von Bauwerken ist in den Eurocodes ein auf Grenzzustandsgleichungen basierendes Nachweiskonzept vorgesehen. Dabei werden die Grenzzustände meist mit semiprobabilistischen Verfahren mithilfe von Teilsicherheitsbeiwerten nachgewiesen. Alternativ dürfen bei Zustimmung der zuständigen Behörde auch probabilistische Verfahren verwendet werden.

Die Grenzzustandsgleichungen werden durch deterministische Funktionen beschrieben, die von verschiedenen

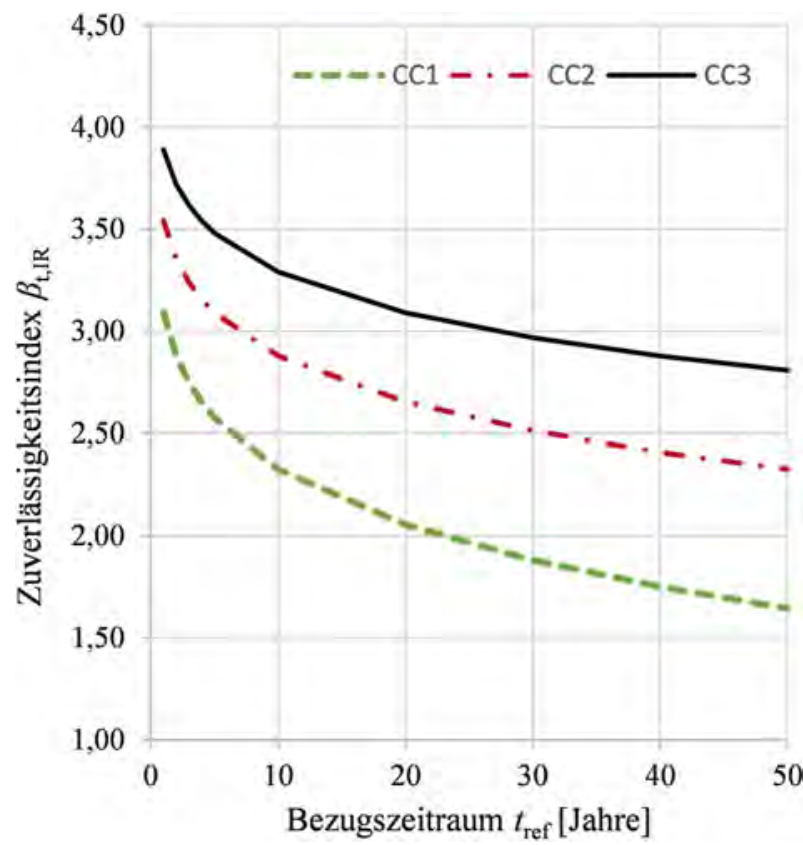

Bild 2 Zuverlässigkeitsindex aus dem Kriterium Einzelrisiko (Individual risk = IR) [1]

Reliability index resulting from individual risk criterion for buildings [1]

physikalischen Eingangsgrößen (Einwirkungen, Materialeigenschaften, geometrische Daten, Modellunsicherheiten), den sog. Basisvariablen, abhängig sind. Ein Bauwerk kann nach EN 1990:2002 [8] als „zuverlässig“ eingestuft werden, sofern kein Grenzzustand überschritten wird. Für den Nachweis sind dabei Bemessungswerte der Basisvariablen zu verwenden. Aus mathematischer Sicht muss somit für jeden Grenzzustand die folgende Bedingung nachgewiesen werden:

$$
g\left(F_{\mathrm{d}}, X_{\mathrm{d}}, a_{\mathrm{d}}, \theta_{\mathrm{d}}\right)=R_{\mathrm{d}}-E_{\mathrm{d}}>0
$$

mit:

$g \quad$ Grenzzustandsfunktion

$F_{\text {d }}$ Bemessungswert für Einwirkungen

$X_{\mathrm{d}}$ Bemessungswert für Materialeigenschaften

$a_{\mathrm{d}} \quad$ Bemessungswert für geometrische Daten

$\theta_{\mathrm{d}} \quad$ Bemessungswert der Variablen zur Berücksichtigung der Modellunsicherheit

$R_{\mathrm{d}} \quad$ Bemessungswert des Widerstands

$E_{\mathrm{d}}$ Bemessungswert der Auswirkung der Einwirkungen

Zur Anpassung der Grenzzustandsfunktionen auf die Besonderheiten der Nachrechnung bestehender Tragwerke sind nach fib Bulletin 80 [1] die folgenden Punkte anzupassen:

- Definition der Bemessungs-/Nachrechnungswerte und Teilsicherheitsbeiwerte;

- Herleitung der „Sicherheitselemente“ unter Berücksichtigung zusätzlicher Informationen aus z.B. Bauwerksuntersuchungen, angepasster Werte für den Zielzuverlässigkeitsindex oder einer Restnutzungsdauer. 
Allgemein kann der Bemessungswert der Auswirkungen von Einwirkungen $E_{\mathrm{d}}$ nach Gl. (4) berechnet werden.

$$
E_{\mathrm{d}}=\gamma_{\mathrm{Sd}} \cdot E\left(\gamma_{\mathrm{f}, \mathrm{i}} \cdot F_{\mathrm{rep}, \mathrm{i}}, a_{\mathrm{d}}\right) i \geq 1
$$

mit:

$\gamma_{S d}$ Teilsicherheitsbeiwert zur Berücksichtigung von Unsicherheiten im Berechnungsmodell der Auswirkungen und im Berechnungsmodell der Einwirkungen

$\gamma_{\mathrm{f}}$ Teilsicherheitsbeiwert, der die Möglichkeit ungünstiger Größenabweichungen der Einwirkungen berücksichtigt

$F_{\text {rep }}$ repräsentativer Wert der Einwirkung

In der Regel kann Gl. (4) nach EN 1990:2002 [8] vereinfacht werden:

$$
E_{\mathrm{d}}=E\left(\gamma_{\mathrm{F}, \mathrm{i}} \cdot F_{\mathrm{rep}, \mathrm{i}}, a_{\mathrm{d}}\right) i \geq 1
$$

mit:

$$
\gamma_{\mathrm{F}, \mathrm{i}}=\gamma_{\mathrm{Sd}} \cdot \gamma_{\mathrm{f}, \mathrm{i}}
$$

Der Bemessungswert der Eigenschaften von Baustoffen und Bauprodukten $X_{\mathrm{d}}$ kann bei linearelastischen Verfahren allgemein nach Gl. (7) berechnet werden.

$$
X_{\mathrm{d}}=\eta \cdot X_{\mathrm{k}} / \gamma_{\mathrm{m}}
$$

mit:

$X_{\mathrm{k}}$ charakteristischer Wert einer Baustoff- oder Produkteigenschaft

$\eta$ Umrechnungsbeiwert zwischen Probeneigenschaften und maßgebenden Eigenschaften im Bauteil

$\gamma_{\mathrm{m}}$ Teilsicherheitsbeiwert für die Baustoff- oder Produkteigenschaft, der Folgendes abdeckt:

- die Möglichkeit ungünstiger Abweichungen der Baustoff- oder Produkteigenschaft vom charakteristischen Wert,

- die Streuung des Umrechnungsbeiwerts $\eta$

Falls die Unsicherheiten des Widerstandsmodells direkt im Bemessungswert $X_{\mathrm{d}}$ integriert werden sollen, kann $\gamma_{\mathrm{m}}$ in Gl. (7) nach EN 1990:2002 [8] durch $\gamma_{M}$ entsprechend Gl. (8) ersetzt werden.

$$
\gamma_{\mathrm{M}}=\gamma_{\mathrm{Rd}} \cdot \gamma_{\mathrm{m}}
$$

mit:

$\gamma_{\text {Rd }}$ Teilsicherheitsbeiwert zur Berücksichtigung von Unsicherheiten des Widerstandsmodells, einschließlich geometrischer Abweichungen, soweit diese nicht explizit berücksichtigt sind

Der Bemessungswert der Tragfähigkeit kann in Abhängigkeit von den Materialeigenschaften $X$ und den geometrischen Abmessungen $a$ bei linearelastischen Verfahren wie folgt beschrieben werden:

$$
R_{\mathrm{d}}=R\left(\eta \cdot X_{\mathrm{k}, \mathrm{i}} / \gamma_{\mathrm{M}, \mathrm{i}} ; a_{\mathrm{d}}\right) \quad i \geq 1
$$

\section{2}

Kalibrierung von Teilsicherheitsbeiwerten

Vorausgesetzt, die Tragfähigkeit $R$ und die Einwirkung $E$ folgen einer Gaußschen Normalverteilung, können die Bemessungswerte $R_{\mathrm{d}}$ und $E_{\mathrm{d}}$ der Variablen $R$ und $E$ unter Berücksichtigung der Grenzzustandsgleichung nach Gl. (3) am FORM-basierten Bemessungspunkt mit den Gln. (10) bzw. (11) berechnet werden.

$$
\begin{aligned}
& R_{\mathrm{d}}=\mu_{\mathrm{R}}-\alpha_{\mathrm{R}} \cdot \beta \cdot \sigma_{\mathrm{R}} \\
& E_{\mathrm{d}}=\mu_{\mathrm{E}}-\alpha_{\mathrm{E}} \cdot \beta \cdot \sigma_{\mathrm{E}}
\end{aligned}
$$

mit:

$\mu$ Mittelwert

$\sigma$ Standardabweichung

$\alpha_{\mathrm{R}}$ Wichtungs-/Sensitivitätsfaktor für die Widerstandsseite

$\alpha_{\mathrm{E}}$ Wichtungs-/Sensitivitätsfaktor für die Einwirkungsseite

Der Wert $\alpha$ ist für ungünstige Einwirkungen oder deren Auswirkungen negativ und für Widerstände positiv. Nach EN 1990:2002 [8] darf für die Bemessung von Neubauwerken $\alpha_{\mathrm{E}}=-0,7$ und $\alpha_{\mathrm{R}}=0,8$ verwendet werden, wenn die Bedingung $0,16 \leq \sigma_{\mathrm{E}} / \sigma_{\mathrm{R}}<7,6$ eingehalten wird. $\sigma_{\mathrm{E}}$ und $\sigma_{\mathrm{R}}$ sind dabei die Standardabweichung der Auswirkung $E$ bzw. des Widerstands $R$.

Liegt das Verhältnis $\sigma_{\mathrm{E}} / \sigma_{\mathrm{R}}$ außerhalb der genannten Grenzen, sollte für die Variable mit der größten Standardabweichung $\alpha= \pm 1$ und für die Variable mit der kleinsten Standardabweichung $\alpha= \pm 0,4$ gewählt werden. Sind Widerstand und Einwirkung abhängig von verschiedenen Zufallsvariablen, gilt $\alpha_{\mathrm{E}}=-0,7$ und $\alpha_{\mathrm{R}}=0,8$ lediglich für die dominante Variable (Variable mit dem größten Einfluss). Für nicht dominante Variablen (alle anderen Variablen) gilt nach EN 1990:2002 [8] $\alpha_{\mathrm{E}}=-0,28$ und $\alpha_{\mathrm{R}}=$ 0,32 .

Auch bei den im fib Bulletin 80 [1] beschriebenen semiprobabilistischen Verfahren wurde mit den in EN 1990:2002 [8] definierten standardisierten Sensitivitätsfaktoren gearbeitet. Ein Vergleich der semiprobabilistischen Verfahren mit probabilistischen Berechnungen ohne festgelegte Sensitivitätsfaktoren in [1] zeigt, dass auch die semiprobabilistischen Verfahren mit den standardisierten Sensitivitätsfaktoren zu konsistenten Ergebnissen führen.

Aufbauend auf den mithilfe der „First Order Reliability Method“ (FORM) bestimmten Bemessungswerten können Gleichungen zur Bestimmung der Teilsicherheitsbeiwerte $\gamma_{\mathrm{m}}$ und $\gamma_{\mathrm{f}}$ angegeben werden. Eine wesentliche Größe zur Beschreibung der Tragfähigkeit ist die Materialfestigkeit, welche meist durch eine Normalverteilung oder eine logarithmische Normalverteilung beschrieben werden kann. Hinweise zur Wahl der Verteilungsfunktion können z. B. [3] entnommen werden. 
Nach fib Bulletin 80 [1] ergeben sich exemplarisch folgende Gleichungen:

$$
\begin{aligned}
& \gamma_{\mathrm{m}}=\frac{X_{\mathrm{k}}}{X_{\mathrm{d}}}=\frac{\mu_{\mathrm{X}}\left(1-1,645 \cdot V_{\mathrm{X}}\right)}{\mu_{\mathrm{X}}\left(1-\alpha_{\mathrm{R}} \cdot \beta \cdot V_{\mathrm{X}}\right)} \quad \text { Normalverteilung } \\
& \gamma_{\mathrm{m}}=\frac{X_{\mathrm{k}}}{X_{\mathrm{d}}}=\frac{\mu_{\mathrm{X}} \exp \left(-1,645 \cdot V_{\mathrm{X}}\right)}{\mu_{\mathrm{X}} \exp \left(-\alpha_{\mathrm{R}} \cdot \beta \cdot V_{\mathrm{X}}\right)} \text { log. (12) } \\
& \text { Gl. (13) }
\end{aligned}
$$

mit:

$V_{\mathrm{X}}$ Variationskoeffizient der Materialeigenschaft

$X_{\mathrm{k}}$ charakteristischer Wert der Materialeigenschaft (hier 5\%-Quantilwert)

Entsprechende Gleichungen zur Bestimmung des Teilsicherheitsbeiwerts $\gamma_{\mathrm{f}}$, getrennt nach ständigen und veränderlichen Einwirkungen, sind im fib Bulletin 80 [1] enthalten.

Eine weitere wesentliche Einflussgröße stellen die Modellunsicherheiten $\theta$ auf Einwirkungs- und Widerstandsseite dar. Werden diese explizit berücksichtigt, gilt für die Grenzzustandsgleichung:

$$
g=\theta_{\mathrm{R}} \cdot R-\theta_{\mathrm{E}} \cdot E
$$

mit:

$\theta_{\mathrm{R}}$ Modellunsicherheit des Widerstandsmodells $\theta_{\mathrm{E}}$ Modellunsicherheit der Einwirkung

Falls den Modellunsicherheiten eine Gaußsche Normalverteilung zugrunde liegt, können nach fib Bulletin 80 [1] die Teilsicherheitsbeiwerte $\gamma_{\text {Rd }}$ und $\gamma_{\text {Sd }}$ wie folgt berechnet werden.

$$
\begin{aligned}
& \gamma_{\mathrm{Rd}}=\frac{\mu_{\theta \mathrm{R}}}{\theta_{\mathrm{Rd}}}=\frac{1}{1-\alpha_{\mathrm{R}} \cdot \beta \cdot V_{\theta \mathrm{R}}} \\
& \gamma_{\mathrm{Sd}}=\frac{\theta_{\mathrm{Ed}}}{\mu_{\theta \mathrm{E}}}=1-\alpha_{\mathrm{E}} \cdot \beta \cdot V_{\theta \mathrm{E}}
\end{aligned}
$$

Darüber hinaus sind im fib Bulletin 80 [1] weitere Ansätze und Hinweise zur Bestimmung der Teilsicherheitsbeiwerte $\gamma_{R d}$ und $\gamma_{S d}$ enthalten.

Für die in den nachfolgenden Abschn. 3.3 und 3.4 beschriebenen Verfahren nach fib Bulletin 80 [1] wurden zur Berechnung der Teilsicherheitsbeiwerte standardisierte Sensitivitätsfaktoren verwendet. Während die „Adjusted partial factor method“ (APFM) auf Anpassungsfaktoren zur Modifikation der in den Eurocodes enthaltenen Teilsicherheitsbeiwerten basiert, werden bei der „Design value method“ (DVM) ausgehend von einem Stufe-II-Verfahren nach EN 1990:2002 [8] direkt Teilsicherheitsbeiwerte für die Nachrechnung abgeleitet. Dadurch können bei der „Design value method“ mehr bauwerkspezifische Informationen berücksichtigt werden.

\subsection{Nachrechnungswertmethode - „Design value method“" (DVM)}

Im vorliegenden Abschnitt wird die Nachrechnung bestehender Tragwerke mit modifizierten Teilsicherheitsbeiwerten basierend auf der „Design value method“ (DVM) nach fib Bulletin 80 [1] vorgestellt. Als wesentliche Grundlage werden dabei die tatsächliche Verteilung der zu betrachtenden Variablen $X$ sowie der Zielzuverlässigkeitsindex verwendet.

Für die Widerstandsseite kann der Nachrechnungswert der Tragfähigkeit $R_{\mathrm{d}}$ mit Gl. (9) bestimmt werden. Der Teilsicherheitsbeiwert $\gamma_{M}$ kann als Produkt nach Gl. (17) berechnet werden. Dabei wurde $\gamma_{\text {Rd }}$ aufgeteilt in die beiden Bestandteile $\gamma_{\text {Rd1 } 1}$ zur Berücksichtigung der Modellunsicherheit und $\gamma_{\mathrm{Rd} 2}$ für geometrische Abweichungen.

$$
\gamma_{\mathrm{M}}=\gamma_{\mathrm{Rd}} \cdot \gamma_{\mathrm{m}}=\gamma_{\mathrm{Rd} 1} \cdot \gamma_{\mathrm{Rd} 2} \cdot \gamma_{\mathrm{m}}
$$

Für gewöhnliche Stahlbetontragwerke wird im fib Bulletin 80 [1] für $\gamma_{\mathrm{Rd} 1}$ für die Betonfestigkeit ein Wert von 1,10 und für Betonstahl ein Wert von 1,025 empfohlen. Für $\gamma_{\text {Rd2 }}$ kann nach [1] für Beton ein Wert von 1,10 und für Bewehrung ein Wert von 1,05 verwendet werden. Kann durch eine qualifizierte Bauwerksuntersuchung eine vernachlässigbare Streuung der geometrischen Größen festgestellt werden, so darf $\gamma_{\mathrm{Rd} 2}=1,0$ verwendet werden.

Darüber hinaus ist im fib Bulletin 80 [1] der Teilsicherheitsbeiwert $\gamma_{R d}$ in Abhängigkeit vom Zielzuverlässigkeitsindex $\beta$ beschrieben. In Bild 3 ist der in Gl. (15) beschriebene Zusammenhang zwischen Zielzuverlässigkeitsindex $\beta$ und $\gamma_{R d}$ dargestellt. Den dargestellten Verläufen liegt dabei eine logarithmische Normalverteilung, $\alpha_{R}=0,32$ (nicht dominant) und $\mu_{\theta \mathrm{R}}=1$ zugrunde.

Die Fälle $V_{\theta \mathrm{R}}=0,14$ für Beton und $V_{\theta \mathrm{R}}=0,06$ für Betonstahl sind dabei bei maßgeblichen geometrischen Unsicherheiten zu verwenden. Hat die Streuung der geometrischen Eigenschaften keinen maßgeblichen Einfluss auf die Tragfähigkeit, so gilt nach fib Bulletin 80 [1] $V_{\theta \mathrm{R}}=0,08$ für Beton und $V_{\theta \mathrm{R}}=0,02$ für Betonstahl.

Über die hier aufgeführten Methoden hinaus sind im Anhang A vom fib Bulletin 80 [1] weitere Hinweise und Ansätze zur Berücksichtigung der Modellunsicherheit enthalten. Neben der Modellunsicherheit spielt der Teilsicherheitsbeiwert für die Baustoff- oder Produkteigenschaft $\gamma_{\mathrm{m}}$ eine wesentliche Rolle. In Bild 4 ist der Teilsicherheitsbeiwert $\gamma_{m}$ in Abhängigkeit vom Variationskoeffizienten $V_{\mathrm{m}}$ unter Annahme einer logarithmischen Normalverteilung nach Gl. (13) sowie $\alpha_{\mathrm{R}}=0,8$ (dominant) für verschiedene Werte des Zielzuverlässigkeitsindex $\beta$ dargestellt.

Entsprechende Verfahren zur Bestimmung der Teilsicherheitsbeiwerte $\gamma_{\mathrm{G}}$ und $\gamma_{\mathrm{Q}}$ für ständige und veränderliche (Nutz-, Schnee-, Wind- und Verkehrslasten) Lasten mit der DVM sind ebenfalls im fib Bulletin 80 [1] enthalten. 


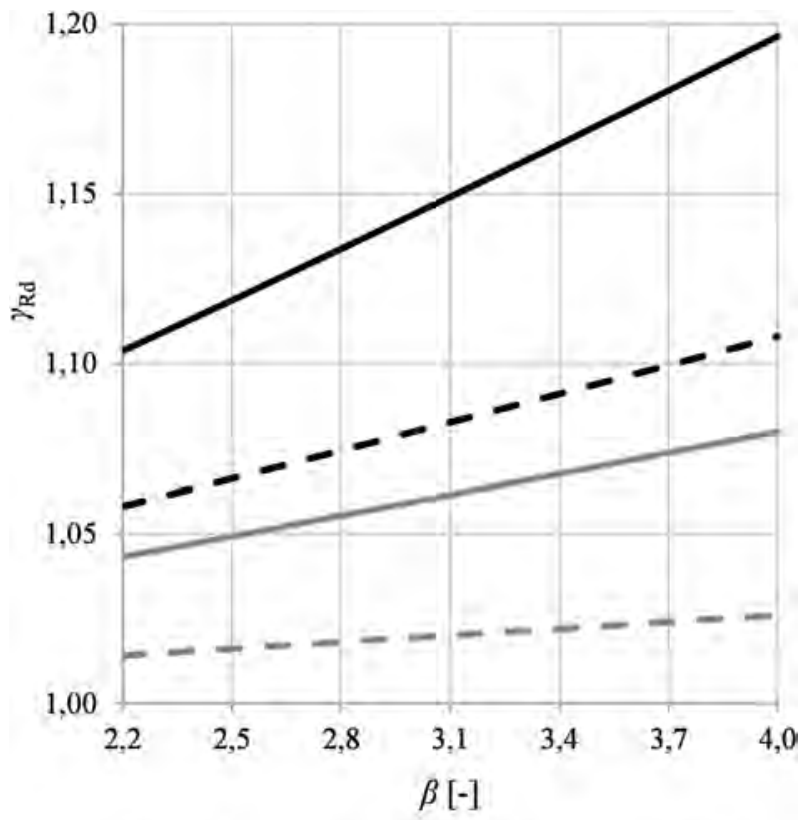

- Beton $V_{\theta \mathrm{R}}=0,14 \quad--$ Beton $V_{\theta \mathrm{R}}=0,08$
Betonstahl $V_{\theta \mathrm{R}}=0,06-$ Betonstahl $V_{\theta \mathrm{R}}=0,02$

Bild 3 Teilsicherheitsbeiwert $\gamma_{R d}$ in Abhängigkeit vom Zielzuverlässigkeitsin$\operatorname{dex} \beta[1]$

Variation of the partial factor $\gamma_{\mathbb{R} d}$ as function of the target reliability $\beta$ [1]

\subsection{Angepasste Teilsicherheitsbeiwertmethode - „Adjusted partial factor method" (APFM)}

Nachfolgend wird die Herleitung angepasster Teilsicherheitsbeiwerte zur Bestimmung von Nachrechnungswerten der Materialeigenschaften bestehender Tragwerke nach der „Adjusted partial factor method“ (APFM) nach fib Bulletin 80 [1] vorgestellt. Im Vergleich zur DVM stellt die APFM ein einfacheres, mit den Eurocodes kompatibles Verfahren dar. Für die Anwendung der APFM müssen weniger probabilistische Parameter festgelegt werden.

Im fib Bulletin 80 [1] sind darüber hinaus entsprechende Verfahren zur Bestimmung angepasster Teilsicherheitsbeiwerte für ständige und veränderliche (Nutz-, Schnee-, Wind- und Verkehrslasten) Lasten enthalten. Den Teilsicherheitsbeiwerten der Materialeigenschaften liegt bei diesem Verfahren der in Gl. (18) beschriebene Zusammenhang zugrunde.

$$
\gamma_{\mathrm{X}}=\omega_{\gamma} \cdot \gamma_{\mathrm{X}, \text { new }}
$$

mit:

\section{$\omega_{\gamma} \quad$ Anpassungsfaktor}

$\gamma_{\mathrm{X}, \text { new }}$ Teilsicherheitsbeiwert für Neubau nach Eurocode

Für die Modellunsicherheiten gilt wie auch schon bei der DVM (vgl. Gl. (17)):

$$
\gamma_{\mathrm{Rd}}=\gamma_{\mathrm{Rd} 1} \cdot \gamma_{\mathrm{Rd} 2}
$$

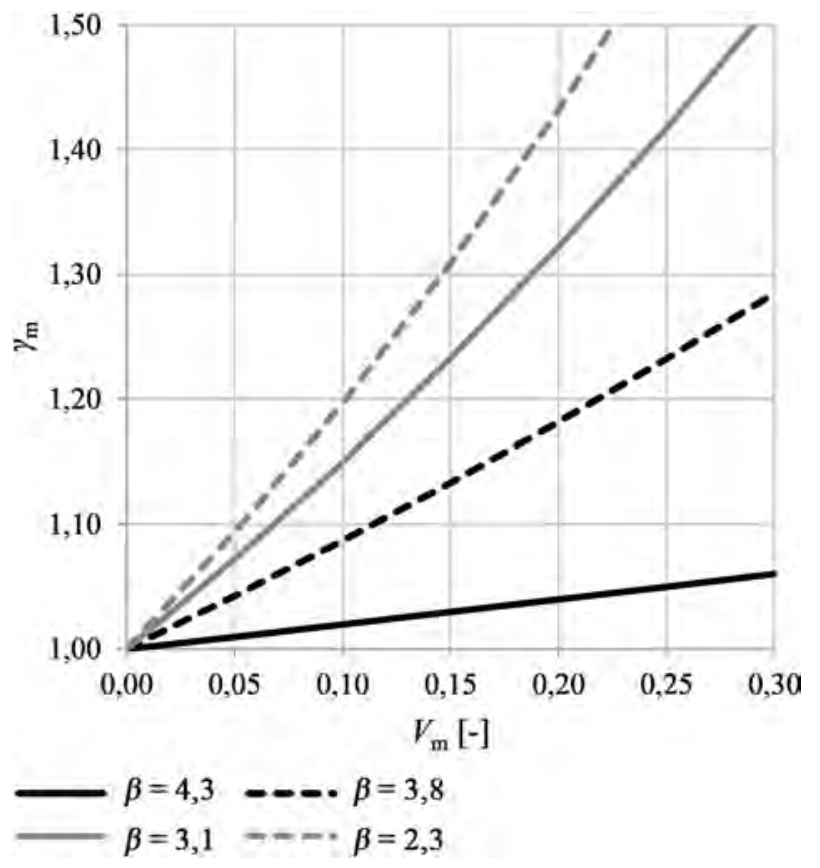

Bild 4 Teilsicherheitsbeiwert $\gamma_{m}$ in Abhängigkeit vom Variationskoeffizienten $V_{\mathrm{m}}[1]$

Variation of the partial factor $\gamma_{m}$ with the coefficient of variation $V_{m}[1]$

Die Werte $\gamma_{\mathrm{Rd} 1}$ und $\gamma_{\mathrm{Rd} 2}$ können z.B. entsprechend der im Abschn. 3.2 beschriebenen Gl. (15) berechnet werden.

Für normalverteilte Größen kann der Anpassungsfaktor mit Gl. (19) bestimmt werden.

$$
\omega_{\gamma}=\frac{\gamma_{\mathrm{Rd}}\left(\beta^{\prime \prime}\right)}{\gamma_{\mathrm{Rd}}\left(\beta^{\prime}\right)} \cdot \frac{1-\alpha_{\mathrm{R}} \cdot \beta^{\prime} \cdot V_{X}^{\prime}}{1-\alpha_{\mathrm{R}} \cdot \beta^{\prime \prime} \cdot V_{X}^{\prime \prime}} \cdot \frac{1-1,645 \cdot V_{X}^{\prime \prime}}{1-1,645 \cdot V_{X}^{\prime}}
$$

Die mit ' gekennzeichneten Parameter beschreiben dabei die dem Neubaufall zugrunde liegenden Annahmen und die mit " gekennzeichneten Parameter beschreiben die für die Nachrechnung anzusetzenden Größen.

Für log-normalverteilte Größen kann der Anpassungsfaktor mit Gl. (20) bestimmt werden.

$$
\begin{array}{r}
\omega_{\gamma}=\frac{\gamma_{\mathrm{Rd}}\left(\beta^{\prime \prime}\right)}{\gamma_{\mathrm{Rd}}\left(\beta^{\prime}\right)} \cdot \exp \left[\alpha_{\mathrm{R}} \cdot\left(\beta^{\prime \prime} \cdot V_{X}^{\prime \prime}-\beta^{\prime} \cdot V_{X}^{\prime}\right)\right. \\
\left.-1,645 \cdot\left(V_{X}^{\prime \prime}-\cdot V_{X}^{\prime}\right)\right]
\end{array}
$$

Falls keine weiteren Informationen vorliegen, kann nach [1] für den Variationskoeffizienten von Beton der im Neubaufall verwendete Wert $V_{C^{\prime}}=0,15$ und für die Bewehrung $V_{\mathrm{S}^{\prime}}=0,05$ angesetzt werden.

In Bild 5 ist der Anpassungsfaktor $\omega_{\gamma}$ für log-normalverteilte Größen nach Gl. (20) unter Annahme eines Zielzuverlässigkeitsindex im Neubaufall $\beta^{\prime}=3,8$ und eines Sensitivitätsfaktors $\alpha_{\mathrm{R}}=0,8$ dargestellt. Zusätzliche Informationen in Hinblick auf den Variationskoeffizienten der Materialeigenschaften sind in Bild 5 nicht berücksichtigt; d.h., es gilt $V_{\mathrm{X}}{ }^{\prime \prime}=V_{\mathrm{X}}$. 


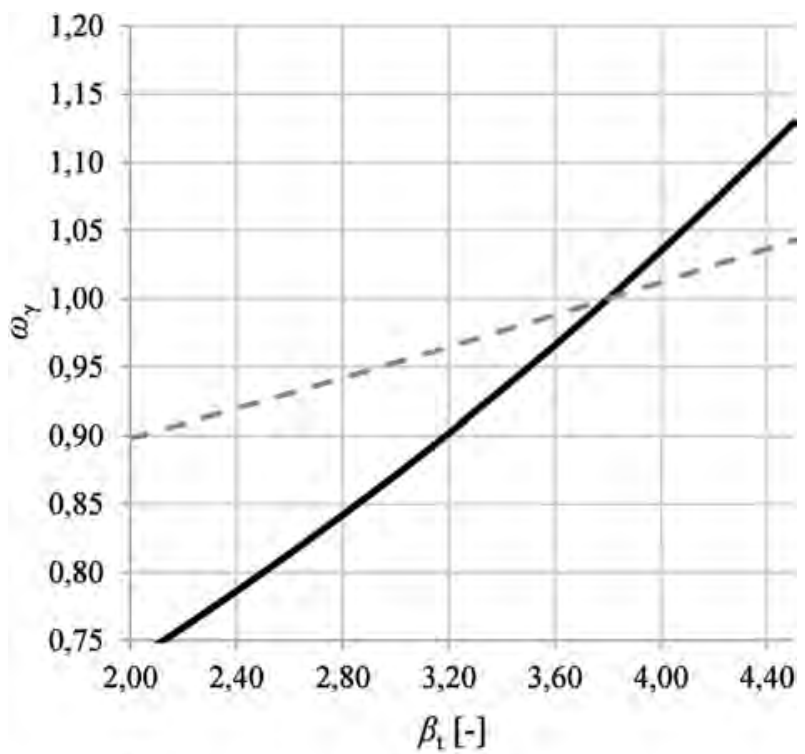

- Beton - - Betonstahl

Bild 5 Einfluss des Zielzuverlässigkeitsindex $\beta_{\mathrm{t}}$ für die Nachrechnung auf den Anpassungsfaktor $\omega_{\gamma}[1]$

Influence of the target reliability index $\beta_{\mathrm{f}}$ for existing structures on the adjustment factor $\omega_{\gamma}[1]$

Tab. 4 Variationskoeffizienten der Modellunsicherheit $V_{\theta R}$ Coefficient of variation of the model uncertainty $V_{\theta R}$

\begin{tabular}{lll}
\hline & Beton & Betonstahl \\
\hline$V_{\theta \mathrm{R}, 1}$ & 0,075 & 0,020 \\
$V_{\theta \mathrm{R}, 2}$ & 0,075 & 0,040 \\
\hline
\end{tabular}

Die Werte $\gamma_{\mathrm{Rd} 1}$ und $\gamma_{\mathrm{Rd} 2}$ wurden gemäß Gl. (15) unter Annahme eines Sensitivitätsfaktors $\alpha_{\mathrm{R}}=0,32$ (nicht dominante Variablen) sowie der in Tab. 4 zusammengefassten Variationskoeffizienten $V_{\theta \mathrm{R}}$ berechnet. Somit ist in Bild 5 lediglich der Einfluss des im Rahmen der Nachrechnung anzusetzenden Zielzuverlässigkeitsindex $\beta^{\prime \prime}=\beta_{\mathrm{t}}$ zu erkennen.

Liegen zusätzliche Informationen über den tatsächlichen Variationskoeffizienten $V^{\prime \prime}$ der betrachteten Materialeigenschaft vor oder weicht der Zielzuverlässigkeitsindex $\beta^{\prime \prime}=\beta_{\mathrm{t}}$ vom der Bemessung zugrunde liegenden Wert ab, können die in Bild 6 für Betonstahl und Bild 7 für Beton angegebenen Werte für den Anpassungsfaktor $\omega_{\gamma}$ nach Gl. (20) verwendet werden. Die dargestellten Verläufe basieren auf einer logarithmischen Normalverteilung, einem Zielzuverlässigkeitsindex im Neubaufall $\beta^{\prime}=3,8$, einem Sensitivitätsfaktor $\alpha_{\mathrm{R}}=0,8$ (dominant), den in Tab. 4 zusammengefassten Werten für den Variationskoeffizienten der Modellunsicherheit (nicht dominant) sowie einem Variationskoeffizienten $V_{S^{\prime}}=0,05$ für Betonstahl und $V_{\mathrm{C}^{\prime}}=0,15$ für Beton.

Bei $V_{\mathrm{S}}^{\prime \prime} / V_{\mathrm{S}^{\prime}}<0,8$ bzw. $V_{\mathrm{C}}^{\prime \prime} / V_{\mathrm{C}}{ }^{\prime}<0,5$ müssen die Anpassungsfaktoren $\omega_{\gamma}$ gesondert berechnet werden, da die Modellunsicherheiten zu dominanten Variablen werden und somit die Bild 6 und Bild 7 zugrunde liegenden Annahmen nicht mehr zutreffen.

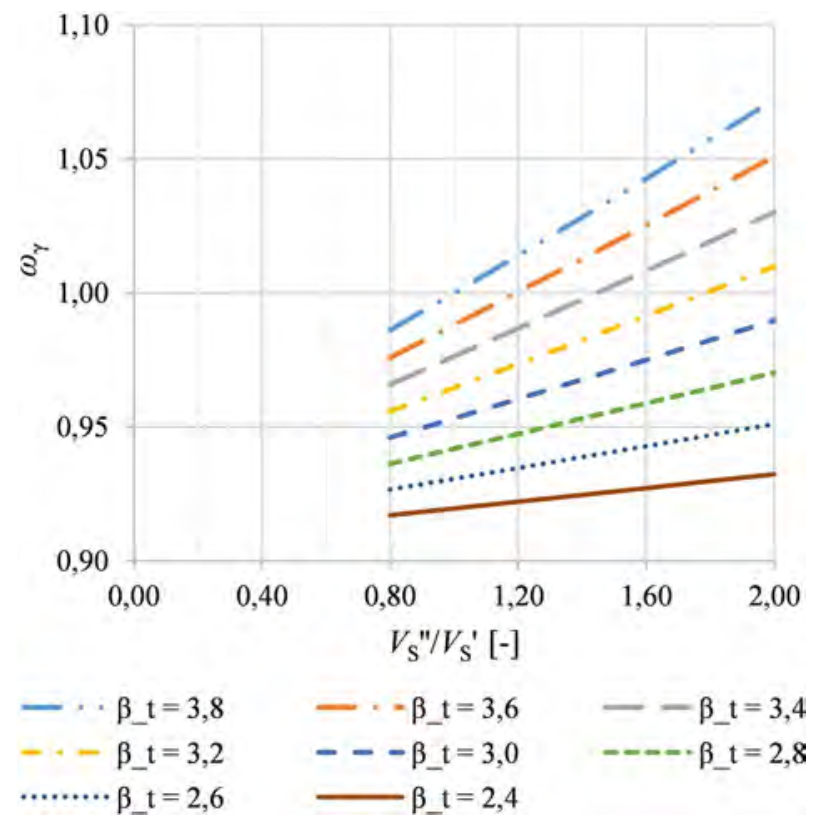

Bild 6 Einfluss des Verhältnisses $V_{S}^{\prime \prime} / V_{S}^{\prime}$ auf den Anpassungsfaktor $\omega_{\gamma}[1]$ Influence of the ratio $V_{S} \prime / / V_{S}^{\prime}$ on the adjustment factor $\omega_{\gamma}[1]$

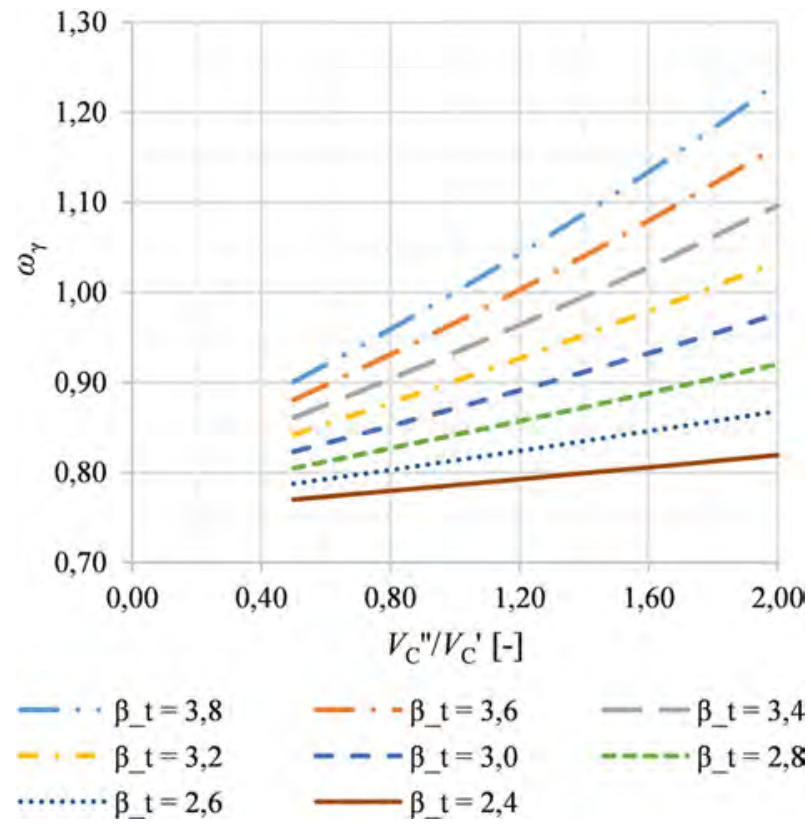

Bild 7 Einfluss des Verhältnisses $V_{C}{ }^{\prime \prime} / V_{\mathcal{C}}$ auf den Anpassungsfaktor $\omega_{\gamma}[1]$ Influence of the ratio $V_{C}{ }^{\prime \prime} / V_{C}{ }^{\prime}$ on the adjustment factor $\omega_{\gamma}[1]$

Weitere Informationen zur „Adjusted partial factor method“, einschließlich der Herleitung von Anpassungsfaktoren $\omega_{\gamma}$ für ständige und veränderliche Einwirkungen, können fib Bulletin 80 [1] entnommen werden.

\section{Anwendungsbeispiel 4.1 Allgemeines}

In diesem Abschnitt wird die Anwendung der „Design value method“ (DVM) und der „Adjusted partial factor method" (APFM) an einem einfachen Beispiel erläutert. Darüber hinaus werden die Unterschiede im Vergleich zu 
einer konventionellen Nachrechnung mit den gemäß Eurocodes für den Neubau beschriebenen Teilsicherheitsbeiwerten dargestellt. Allgemein werden im vorliegenden Beispiel lediglich die materialspezifischen Teilsicherheitsbeiwerte der Widerstandsseite betrachtet. Ein weiteres Beispiel, welches auch die Teilsicherheitsbeiwerte der Einwirkungsseite beinhaltet, ist im fib Bulletin 80 [1] enthalten.

Im gewählten Beispiel ist aufgrund der mit einer Umnutzung einhergehenden Lasterhöhung eine Nachrechnung erforderlich. Da keine Informationen über die tatsächlichen Materialeigenschaften vorliegen, sind diese im Rahmen einer Bestandsaufnahme zu ermitteln.

\subsection{Ergebnisse der Bauwerksuntersuchung - Materialkenndaten}

Zur Bestimmung der In-situ-Betondruckfestigkeit wurden nach DIN EN 13791/A20:2017-02 [14] und DAfStb-Heft 619 [3] aus dem Bauwerk fünf Bohrkerne mit einem Durchmesser von $100 \mathrm{~mm}$ entnommen. Aus diesen Bohrkernen wurden Probekörper mit einer Schlankheit $\lambda=$ $h / \phi=1,0$ vorbereitet, an denen die Betondruckfestigkeit bestimmt wurde. Die Ergebnisse sind in Tab. 5 zusammengefasst. Aufgrund der verwendeten Probekörpergeometrie können die ermittelten Werte der Betondruckfestigkeit mit den an Würfeln mit einer Kantenlänge von $150 \mathrm{~mm}$ geprüften Werten gleichgesetzt werden.

Für eine auf den Eurocodes basierende Nachrechnung muss die an den Bohrkernen ermittelte Festigkeit noch auf die entsprechende Zylinderdruckfestigkeit $f_{\text {ci,cyl150/300 }}$ umgerechnet werden [3].

Aus den in Tab. 5 aufgelisteten Werten folgen die in Tab. 6 zusammengefassten Parameter der Stichprobe. Hierbei ist $\mathrm{zu}$ beachten, dass aus statistischer Sicht ein größerer Stichprobenumfang zu empfehlen ist, um Unsicherheiten $\mathrm{zu}$ reduzieren.

Der statistischen Auswertung liegt eine logarithmische Normalverteilung nach dem in EN 1990:2002 - An-

Tab. 5 Ergebnisse der Betondruckfestigkeitsuntersuchung durch Bohrkernentnahme $\left[\mathrm{N} / \mathrm{mm}^{2}\right]$

Investigation of the actual concrete compressive strength by drill core testing $\left[\mathrm{N} / \mathrm{mm}^{2}\right]$

\begin{tabular}{llllll}
\hline & BK 1 & BK 2 & BK 3 & BK 4 & BK 5 \\
\hline$f_{\text {ci,BK,is }}$ & 29,4 & 27,4 & 27,8 & 25,7 & 32,1 \\
$f_{\text {ci,cyl150/300,is }}$ & 24,1 & 22,5 & 22,8 & 21,1 & 26,3 \\
\hline
\end{tabular}

Tab. 6 Parameter der Stichprobe Parameters of the sample

\begin{tabular}{llll}
\hline Mittelwert & $m_{\mathrm{x}}$ & {$\left[\mathrm{N} / \mathrm{mm}^{2}\right]$} & 23,4 \\
Standardabweichung & $s_{\mathrm{X}}$ & {$\left[\mathrm{N} / \mathrm{mm}^{2}\right]$} & 1,96 \\
Variationskoeffizient & $v_{\mathrm{x}}$ & {$[-]$} & 0,08 \\
\hline
\end{tabular}

hang D [8] beschriebenen Bayes'schen Verfahren mit der Annahme „,,$_{\mathrm{x}}$ unbekannt“ zugrunde. Der charakteristische 5\%-Quantilwert der In-situ-Betondruckfestigkeit berechnet sich somit gemäß Gl. (21) mit $k_{\mathrm{n}=5}=2,33 \mathrm{zu}$ $x_{\mathrm{k}}=19,2 \mathrm{~N} / \mathrm{mm}^{2}$.

$$
x_{\mathrm{k}}=\exp \left(m_{\mathrm{y}}-k_{\mathrm{n}} \cdot s_{\mathrm{y}}\right)
$$

mit:

$m_{\gamma}$ Mittelwert der logarithmierten Einzelwerte

$$
m_{\mathrm{y}}=\frac{1}{n} \sum \ln \left(x_{\mathrm{i}}\right)
$$

$s_{\gamma} \quad$ Standardabweichung der logarithmierten Einzelwerte

$$
s_{\mathrm{y}}=\sqrt{\frac{1}{n-1} \Sigma\left(\ln x_{\mathrm{i}}-m_{\mathrm{y}}\right)^{2}}
$$

$k_{\mathrm{n}} \quad$ Fraktilenfaktor zur Bestimmung charakteristischer Werte nach [8]

In Tab. 7 und Tab. 8 sind die entsprechenden Ergebnisse der Betonstahluntersuchungen dargestellt. Unter Annahme einer logarithmischen Normalverteilung kann mit Gl. (21) mit $k_{\mathrm{n}=4}=2,63$ ein charakteristischer 5\%-Quantilwert der Streckgrenze von $394,6 \mathrm{~N} / \mathrm{mm}^{2}$ berechnet werden.

\subsection{Bestimmung von Teilsicherheitsbeiwerten für die Nachrechnung}

Unter Berücksichtigung des in Abschn. 2.3 beschriebenen Verfahrens zur Festlegung des Zielzuverlässigkeitsindex für die Nachrechnung wurde hier exemplarisch für die Schadensfolgeklasse CC 2 und einen Bezugszeitraum von 13 Jahren nach Bild 2 ein Wert $\beta_{\mathrm{t}}=2,8$ gewählt. Grundsätzlich sind im Einzelfall alle jeweils zulässigen Verfahren zur Bestimmung des Zielzuverlässigkeitsindex $\mathrm{zu}$ überprüfen, um den maßgebenden Wert festzulegen.

Als Variationskoeffizienten der jeweiligen Materialeigenschaft wurden die an der Stichprobe ermittelten Werte gemäß Tab. 6 und Tab. 8 verwendet. Die daraus nach der DVM bzw. APFM resultierenden modifizierten Teilsicherheitsbeiwerte bzw. Anpassungsfaktoren sowie die nach DIN EN 1992-1-1 [15] anzusetzenden Werte sind in Tab. 9 und Tab. 10 zusammengefasst.

Tab. 7 Ergebnisse der Betonstahluntersuchungen $\left[\mathrm{N} / \mathrm{mm}^{2}\right]$ Investigation of the actual mechanical properties of steel $\left[\mathrm{N} / \mathrm{mm}^{2}\right]$

\begin{tabular}{lllll}
\hline & $\mathbf{1}$ & $\mathbf{2}$ & $\mathbf{3}$ & $\mathbf{4}$ \\
\hline$f_{\mathrm{yi}, \text { is }}$ & 445,4 & 431,7 & 416,5 & 452,6 \\
\hline
\end{tabular}

Tab. 8 Parameter der Stichprobe Parameters of the sample

\begin{tabular}{llll}
\hline Mittelwert & $m_{\mathrm{x}}$ & {$\left[\mathrm{N} / \mathrm{mm}^{2}\right]$} & 436,6 \\
Standardabweichung & $s_{\mathrm{X}}$ & {$\left[\mathrm{N} / \mathrm{mm}^{2}\right]$} & 15,93 \\
Variationskoeffizient & $v_{\mathrm{X}}$ & {$[-]$} & 0,04 \\
\hline
\end{tabular}


Tab. 9 Teilsicherheitsbeiwerte für die Nachrechnung des bestehenden Tragwerks nach der DVM

Partial factors for the assessment of the existing structure based on the DVM

\begin{tabular}{lllll}
\hline & $\begin{array}{l}\text { DVM } \\
\boldsymbol{\beta}_{\mathrm{t}}=\mathbf{2 , \mathbf { 8 }}\end{array}$ & $\begin{array}{l}\text { DVM } \\
\boldsymbol{\beta}_{\mathrm{t}}=\mathbf{3 , 8}\end{array}$ & Quelle & DIN EN 1992 \\
\hline$\gamma_{\mathrm{c}}{ }^{1)}$ & 1,05 & 1,12 & Bild 4 & - \\
$\gamma_{\mathrm{Rd}, \mathrm{c}}{ }^{2)}$ & 1,13 & 1,19 & Bild 3 & - \\
$\gamma_{\mathrm{C}}$ & $\mathbf{1 , 1 9}$ & $\mathbf{1 , 3 3}$ & Gl. $(17)$ & $\mathbf{1 , 5 0}$ \\
$\gamma_{\mathrm{S}}{ }^{1)}$ & 1,02 & 1,06 & Bild 4 & - \\
$\gamma_{\mathrm{Rd}, \mathrm{s}}{ }^{3)}$ & 1,06 & 1,08 & Bild 3 & - \\
$\gamma_{\mathrm{S}}$ & $\mathbf{1 , 0 8}$ & $\mathbf{1 , 1 4}$ & Gl. $(17)$ & $\mathbf{1 , 1 5}$ \\
\hline
\end{tabular}

Annahmen:

1) logarithmische Normalverteilung; $\alpha_{\mathrm{R}}=0,80$

2) logarithmische Normalverteilung; $\alpha_{\mathrm{R}}=0,32 ; V \theta_{\mathrm{R}}=0,14$

3) logarithmische Normalverteilung; $\alpha_{\mathrm{R}}=0,32 ; V \theta_{\mathrm{R}}=0,06$

Darüber hinaus sind in Tab. 9 und Tab. 10 zum Vergleich die modifizierten Teilsicherheitsbeiwerte für den in Deutschland gebräuchlichen Wert des Zielzuverlässigkeitsindex $\beta_{\mathrm{t}}=3,8$ angegeben. Bei diesen Werten wird somit lediglich die im Vergleich zum Neubau günstigere, tatsächlich am Bauwerk vorliegende Streuung der Materialeigenschaften berücksichtigt.

\subsection{Vergleich mit einer konventionellen Nachrechnung nach DIN EN 1992-1-1}

Durch die Berücksichtigung der tatsächlich am Bauwerk vorliegenden Materialstreuungen kann sowohl mit der DVM als auch mit der APFM eine deutliche Reduzierung der Teilsicherheitsbeiwerte erreicht werden. Im vorliegenden Fall liegen die tatsächlichen Streuungen unterhalb derer, die den Teilsicherheitsbeiwerten im Neubaufall nach Eurocodes zugrunde liegen. Zudem kann durch eine Absenkung des Zielzuverlässigkeitsindex eine weitere Reduzierung der Teilsicherheitsbeiwerte erfolgen.

Im Vergleich zu den nach DIN EN 1992-1-1 [15] anzusetzenden Teilsicherheitsbeiwerten kann im vorliegenden Beispiel der Teilsicherheitsbeiwert $\gamma_{C}$ zur Bestimmung des Nachrechnungswerts der Betondruckfestigkeit mit der DVM um 21\% und mit der APFM um 19\% reduziert werden. Der Teilsicherheitsbeiwert $\gamma_{S}$ zur Bestimmung des Nachrechnungswerts der Betonstahlstreckgrenze kann mit beiden Verfahren um $6 \%$ reduziert werden.

\section{5}

\section{Zusammenfassung}

Im fib Bulletin 80 [1] werden mit der „Design value method“ (DVM) und der „Adjusted partial factor method“ (APFM) zwei alternative Verfahren zur Bestimmung modifizierter Teilsicherheitsbeiwerte für die Nachrechnung von bestehenden Tragwerken vorgestellt. Beide Verfahren sind kompatibel mit dem Sicherheitskonzept der Eu-
Tab. 10 Teilsicherheitsbeiwerte für die Nachrechnung des bestehenden Tragwerks nach der APFM

Partial factors for the assessment of the existing structure based on the APFM

\begin{tabular}{lllll}
\hline & $\begin{array}{l}\text { APFM } \\
\boldsymbol{\beta}_{\mathrm{t}}=\mathbf{2 , 8}\end{array}$ & $\begin{array}{l}\text { APFM } \\
\boldsymbol{\beta}_{\mathrm{t}}=\mathbf{3 , 8}\end{array}$ & Quelle & DIN EN 1992 \\
\hline$\omega_{\gamma, \mathrm{C}}{ }^{1)}$ & 0,81 & 0,91 & Bild 7 & - \\
$\gamma_{\mathrm{C}}$ & $\mathbf{1 , 2 2}$ & $\mathbf{1 , 3 7}$ & Gl. $(18)$ & $\mathbf{1 , 5 0}$ \\
$\omega_{\gamma, \mathrm{S}^{1)}}$ & 0,94 & 0,99 & Bild 6 & - \\
$\gamma_{\mathrm{S}}$ & $\mathbf{1 , 0 8}$ & $\mathbf{1 , 1 4}$ & Gl. $(18)$ & $\mathbf{1 , 1 5}$ \\
\hline
\end{tabular}

Annahmen:

1) logarithmische Normalverteilung; Zielzuverlässigkeitsindex im Neubaufall $\beta^{\prime \prime}=$ 3,8; $\alpha_{\mathrm{R}}=0,80 ;$ Modellunsicherheiten nach Tab. $4 ; V_{\mathrm{S}}{ }^{\prime}=0,05$ für Betonstahl und $V_{\mathrm{C}^{\prime}}=0,15$ für Beton

rocodes. Zusätzlich können bei beiden Verfahren angepasste Werte des Zielzuverlässigkeitsindex sowie zusätzliche Informationen aus Bauwerksuntersuchungen mithilfe der tatsächlichen Variationskoeffizienten der Materialeigenschaft berücksichtigt werden. Darüber hinaus wird in [1] die Anpassung der Nachrechnung an verschiedene Bezugszeiträume erläutert.

Nicht zuletzt spiegelt sich dies, wie auch im vorliegenden Anwendungsbeispiel zu erkennen, meist in einer Reduktion der Teilsicherheitsbeiwerte wider. Auch ohne eine Modifikation des Zielzuverlässigkeitsindex können durch eine Berücksichtigung der tatsächlich am Bauwerk vorliegenden Streuung der Materialeigenschaften die für die Nachrechnung anzusetzenden Teilsicherheitsbeiwerte entsprechend angepasst werden. Sofern die tatsächliche Streuung der Materialeigenschaften den der Bemessung im Neubaufall zugrunde liegenden Wert unterschreitet, führt dies bei konstanter Zielzuverlässigkeit zu einer Reduzierung der Teilsicherheitsbeiwerte.

Somit können mit den im fib Bulletin 80 [1] vorgestellten Verfahren insbesondere auch sehr konservative Nachrechnungen und damit oft einhergehende hohe Kosten für Verstärkungsmaßnahmen in den meisten Fällen vermieden werden.

Zur Bewertung der beiden Verfahren sind in [1] probabilistische Vergleichsrechnungen enthalten, die zeigen, dass durch die Berücksichtigung zusätzlicher Informationen aus z.B. Bauwerksuntersuchungen mit beiden Verfahren festgelegte Werte des Zuverlässigkeitsindex genauer erreicht werden können als bei einer Verwendung der in den Eurocodes enthaltenen Teilsicherheitsbeiwerte.

Zur Anpassung des Zielzuverlässigkeitsindex werden im fib Bulletin 80 [1] aufbauend auf Schadensfolgeklassen neben Verfahren zur wirtschaftlichen Optimierung auch Verfahren zur Anpassung des Zielzuverlässigkeitsindex an eine verkürzte Restnutzungsdauer vorgestellt.

Inwieweit für die Nachrechnung von Bestandstragwerken eine Anpassung des Zielzuverlässigkeitsindex $\beta$ im bauaufsichtlich geregelten Bereich Berücksichtigung 
finden soll, ist auf nationaler Ebene noch zu entscheiden. Die aktuellen, von CEN/TC 250/WG2.T1 erarbeiteten Entwürfe zur Fortschreibung von Eurocode 0 auf bestehende Tragwerke sehen hierzu jeweils nationale Festlegungen vor.

Die im fib Bulletin 80 [1] aufgeführten Ansätze stellen somit eine wertvolle Grundlage für die anstehende Fachdiskussion auf nationaler Ebene dar.

\section{Literatur}

[1] fib Bulletin 80 - Partial factor methods for existing concrete structures. Recommendation, fib Task Group 3.1. Fédération international du béton (fib), 2016, ISBN 978-2-88394120-5.

[2] DAfStb-Heft 616: Sachstandbericht Bauen im Bestand Teil I „Mechanische Kennwerte historischer Betone, Betonstähle und Spannstähle für die Nachrechnung von bestehenden Bauwerken“. Berlin: Beuth Verlag, 2016.

[3] DAfStb-Heft 619: Sachstandbericht Bauen im Bestand Teil II „Bestimmung charakteristischer Betondruckfestigkeiten". Berlin: Beuth Verlag, 2017.

[4] DBV-Merkblatt „Bauen im Bestand - Beton und Betonstahl“. Eigenverlag, Ausgabe März 2016.

[5] DBV-Merkblatt „Bauen im Bestand - Bewertung der Insitu-Druckfestigkeit von Beton". Eigenverlag, Ausgabe März 2016.

[6] Bundesministerium für Verkehr, Bau und Stadtentwicklung: Richtlinie zur Nachrechnung von Straßenbrücken im Bestand (Nachrechnungsrichtlinie). Berlin, Ausgabe 05/2011.

[7] DBV-Merkblatt „Bauen im Bestand - Modifizierte Teilsicherheitsbeiwerte für Stahlbetonbauteile“. Eigenverlag, Ausgabe März 2013.

[8] EN 1990:2002: Eurocode - Basis of structural design.

[9] DBV-Heft 24: DBV-Heft 24: Schnell, J.; GrünberG, J. STAUder, F.; Fischer, A.: „Begründung eines reduzierten Zuverlässigkeitsindexes und modifizierter Teilsicherheitsbeiwerte für Stahlbetontragwerke im Bestand“. Eigenverlag, Berlin, 2014.

[10] ISO 2394:1998: General principles on reliability for structures.

[11] Steenbergen, R.D.J.M.; VRouwenvelder, A.C.W.M.: Safety philosophy for existing structures and partial factors for traffic load on bridges. Heron, Vol. 55, No. 2, 2010, S. 123-140.

[12] ElduKaIR, Z.A.; Ayyub, B.M.: Analysis of recent U.S. structural and construction failures. Journal of Performance of Constructed Facilities 5 (1991), No. 1, S. 57-73.

[13] STAUDER, F.: Zuverlässigkeitskonzept für bestehende Tragwerke im Wasserbau. Dissertation, Fachgebiet Massivbau und Baukonstruktion, Technische Universität Kaiserslautern, 2015

[14] DIN EN 13791/A20:2017-02: Bewertung der Druckfestigkeit von Beton in Bauwerken oder in Bauwerksteilen; Änderung $A 20$.

[15] DIN EN 1992-1-1:2011-01: Eurocode 2: Bemessung und Konstruktion von Stahlbeton- und Spannbetontragwerken - Teil 1-1: Allgemeine Bemessungsregeln und Regeln für den Hochbau; Deutsche Fassung EN 1992-1-1:2004 + AC:2010 mit DIN EN 1992-1-1/NA:2013-04: Nationaler Anhang; DIN EN 1992-1-1/A1:2015-03: A1-Änderung und DIN EN 1992-1-1/NA/A1:2015-12: A1 Änderung Nationaler Anhang.

\section{Dank}

Ein besonderer Dank gilt allen Mitgliedern der fib Task Group 3.1 für die Erarbeitung des neuen fib Bulletin 80 . Die Task Group entstand im Jahr 2015 aus der früheren Working Group 2A der fib Special Activity Group 7.

Darüber hinaus bedanken sich die Autoren bei dem Forschungsschwerpunkt HiPerCon, der begleitende Arbeiten an der TU Kaiserslautern finanziell unterstützt.

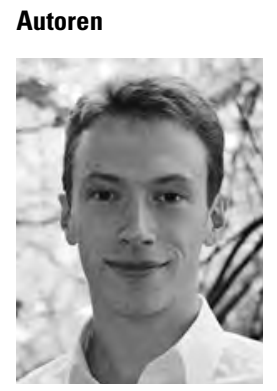

Dipl.-Ing. Michael Weber

Technische Universität Kaiserslautern Fachgebiet Massivbau und Baukonstruktion Paul-Ehrlich-Straße 14 67663 Kaiserslautern michael.weber@bauing.uni-kl.de

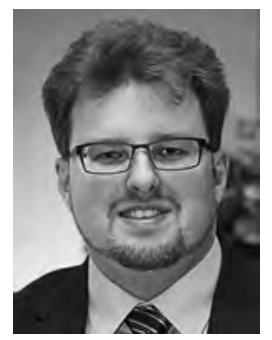

Prof. Dr.-Ing. Robby Caspeele Ghent University, Belgien Department of Structural Engineering Technologiepark-Zwijnaarde 904 B-9052 Ghent, Belgien robby.caspeele@UGent.be

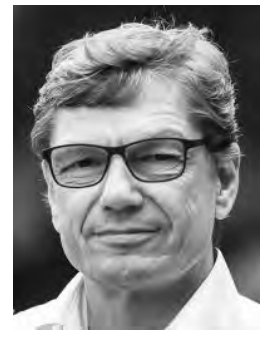

Prof. Dr.-Ing. Jürgen Schnell Technische Universität Kaiserslautern Fachgebiet Massivbau und Baukonstruktion Paul-Ehrlich-Straße 14 67663 Kaiserslautern juergen.schnell@bauing.uni-kl.de

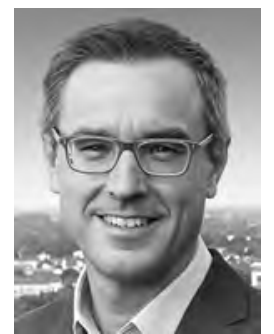

Prof. Dr.-Ing. Christian Glock Technische Universität Kaiserslautern Fachgebiet Massivbau und Baukonstruktion Paul-Ehrlich-Straße 14 67663 Kaiserslautern christian.glock@bauing.uni-kl.de

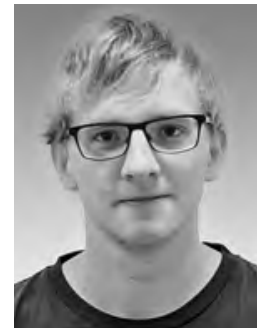

Dr.-Ing. Wouter Botte Ghent University, Belgien Department of Structural Engineering Technologiepark-Zwijnaarde 904 B-9052 Ghent, Belgien wouter.botte@UGent.be 